\title{
A Medical Report Feature for OSSE Rare Disease Registries
}

\author{
Katja SCHUELER ${ }^{\mathrm{a}, 1}$, Axel ZIESCHANK ${ }^{\mathrm{a}}$, Jens GÖBEL ${ }^{\mathrm{a}}$, Jessica VASSEUR ${ }^{\mathrm{a}}$, \\ Jannik SCHAAF ${ }^{\mathrm{a}}$, Katharina HOHENFELLNER ${ }^{\mathrm{b}}$, \\ Sarah WILDENHAIN ${ }^{c}$ and Holger STORF ${ }^{\mathrm{a}}$ \\ a Medical Informatics Group, University Hospital Frankfurt, Germany \\ ${ }^{\mathrm{b}}$ RoMed Klinikum Rosenheim, Germany \\ ${ }^{\mathrm{c}}$ LIBERTAMED GmbH, Germany
}

\begin{abstract}
Web-based patient registries support clinicians by providing a way to effectively store and process data. Here, we present a new feature for the opensource registry software OSSE: medical reports generated with R Markdown. As part of a rare disease research project, we describe the process from requirements assessment to the current state of technical implementation. The feature offers clinicians the possibility to download customised as well as generic reports from an OSSE rare disease registry.
\end{abstract}

Keywords. Medical report, R Markdown, OSSE registry, rare disease, Cystinosis

\section{Introduction}

Web-based patient registries support healthcare by means of effectively storing and sharing patient data. OSSE, an open-source patient registry software developed for research on rare diseases [1] [2], represents a framework for clinicians and scientists to create disease-specific patient registries. During medical research collaborations, we have identified a need among clinicians not only to enter data into OSSE registers in a one-way process, but also to extract data during their clinical routine. Therefore, we have developed, and present here, a medical report feature for OSSE registries. This work is part of an ongoing research project on the nephrotic rare disease Cystinosis [3].

\section{Methods}

We developed the OSSE medical report feature during SAVE, an OSSE registry project on Cystinosis. SAVE serves as a tool to support the standardized documentation of patients during the Cystinosis Outpatient Consultation where patients can consult up to 13 medical specialists within one day [4]. We employed a step-wise procedure to assess software requirements from a multi-disciplinary team of Cystinosis experts from the SAVE project. They provided us with example doctor's letters as well as report samples (.xlsx tables) for each OSSE web form representing the desired structure of the SAVE

${ }^{1}$ Corresponding author, Dr Katja Schueler, Medical Informatics Group, University Hospital Frankfurt, Theodor-Stern-Kai 7, 60596 Frankfurt am Main, Germany; E-mail: katja.schueler@kgu.de 
registry. During recurrent meetings and interviews, we further refined these with the aim of mapping their content to specific variables within the OSSE registry database. After researching for suitable formatting tools, we chose the R package $R$ Markdown [5] [6], because it allowed us to select from a variety of different output formats and to include statistical analysis in medical reports. To establish communication between Java-based OSSE and a running instance of $\mathrm{R}$, which is required to execute $\mathrm{R}$ Markdown, we used the Java library RCaller [7].

\section{Results}

Based on the requirements analysis, we developed two types of medical reports: a) customized medical reports that are able to combine data from the entire OSSE registry database and include customizable additions such as letterhead, narrative text, logos, tables, and formatting; and b) generic medical reports, which automatically output all data of the currently displayed registry form. Both reports are available for download as Word documents (.docx format) in OSSE. Currently, the OSSE medical report feature has been integrated in SAVE and is being tested in practice during the Cystinosis Outpatient Consultation. Two customized reports gather patient data from the entire OSSE registry: one focusing on diagnostic information and the other providing a base of the formal medical report.

\section{Discussion}

The OSSE medical report feature allows clinicians to download data from OSSE registries as formatted documents to facilitate their clinical routine. Customised reports offer the clinician numerous options for data aggregation or integration into existing documentation, but come at the cost of time-consuming creation. Vice versa applies to generic reports. We offered both in an attempt to best cover clinicians' needs. Future research aims at conducting user tests and including international formatting standards.

\section{Conclusion}

This feature allows clinicians to download medical reports from OSSE registries.

\section{References}

[1] Muscholl M, et al. OSSE - Open Source Registry Software Solution, Orphanet J Rare Diseases. $2014 ; 9$.

[2] Storf H, Schaaf J, Kadioglu D, Göbel J, Wagner TOF, and Ückert F, Register für seltene Erkrankungen, Bundesgesundheitsbl. 2017; 60.

[3] Gahl WA, Thoene JG, and Schneider JA, Cystinosis, N Engl J Med. 2002; 347: 111-121.

[4] Hohenfellner K and Deerberg-Wittram J, Coordinated, Cost-effective Care for Rare Disease: The Cystinosis Outpatient Consultation Program at RoMed, NEJM Catalyst. 2020; 1.

[5] R Core Team, R: A Language and Environment for Statistical Computing, R Foundation for Statistical Computing, Vienna, Austria. 2020.

[6] Allaire JJ, et al. Rmarkdown: Dynamic Documents for R. 2020.

[7] Satman M, RCaller: A Software Library for Calling R from Java, BJMCS. 2014; 4. 